\title{
Effects of Lower-limb Muscle Fatigue, Cardiopulmonary Fatigue, and Brain FatigueTasks on One-legged Landing Motion
}

\author{
Yukihiro Higo, MS, RPT and Hironobu KuRUMA, PhD, RPT \\ Faculty of Health Sciences, Graduate School of Human Health Sciences, Tokyo Metropolitan University, Japan
}

\begin{abstract}
Objective: Anterior cruciate ligament (ACL) injury is one of the most frequent sportsinjuries, and previous studies have shown that fatigue is a risk factor for sports injuries. This study aimed to inform prevention of ACL injury by investigating how exercise and desk tasks affect trunk and lower limb alignment and ground reaction force (GRF) during one-legged landing movements. Methods: The study subjects were 12 men who performed a one-legged landing movement from a 30-cm platform before and after fatigue tasks, including lower-limb muscle fatigue, cardiopulmonary fatigue, and brain fatigue tasks. For the measurement of joint angles and moments and GRF, a three-dimensional motion analysis device and a floor reaction-force meter were used. Statistics were performed using Wilcoxon's signed rank sum test as a multiple comparison test with Bonferroni adjustment to compare the difference in effects. Results: The maximum trunk flexion angle during landing on one leg was significantly lower in the brain fatigue group than in the control group. The time to peak vertical GRF (pGRF) was significantly shorter in the leg-muscle fatigue group than in the control group. Conclusion: Brain fatigue may have altered the postural strategy before and after landing, resulting in a decrease in trunk flexion angle. Time to pVGRF was shortened in the leg muscle fatigue group, suggesting that there may be an increased risk of ACL injury. Time to pVGRF during lower extremity muscle fatigue and trunk flexion angle during brain fatigue may be more pronounced during actual sports activities.
\end{abstract}

Key words: ACL injury, Jump-landing motion, Fatigue, Prevention

(Phys Ther Res 00: 00-00, 0000)

\footnotetext{
A crior ciate ligament (ACL) injury is one of the most common sports injuries. ACL injury requires ligament reconstruction surgery for athletes to continue sporting activities ${ }^{1)}$ and may reduce athletic performance and activity. Among athletes and the general public, ACL injury has been associated with early-onset knee osteoarthritis, damage to the meniscus and cartilage surface, and economic loss due to difficulty in working ${ }^{2}$.

Although the mechanism of ACL injury varies by

Received: February 19, 2021

Accepted: August 25, 2021

Advance Publication by J-STAGE: October 27, 2021

Correspondence to: Yukihiro Higo, Faculty of Health Sciences, Graduate School of Human Health Sciences, Tokyo Metropolitan University, Japan, 7-2-10 Higashi-Ogu, Arakawa, Tokyo 116-8551, Japan

\# e-mail: higo.yukihiro@nihon-u.ac.jp

doi: 10.1298/ptr.E10104
}

sport, $70 \%$ result from non-contact injuries ${ }^{3)}$. The injury is commonly caused when changing direction, landing, or decelerating/stopping. The landing position during injury is often a result of loss of balance, backward center of gravity, insufficient knee flexion, and valgus stress of the knee joint $^{3)}$. Recent findings regarding ACL injury mechanisms and dynamic alignment have informed ACL injury prevention strategies. Of non-contact injuries, 58-61\% occur during a jump-landing motion ${ }^{4}$, and athletes with ACL injury had approximately 20\% higher vertical ground reaction force (VGRF) on landing than non-injured athletes ${ }^{5)}$. The posterior GRF (PGRF) was significantly positively correlated with VGRF and the anterior withdrawal force of the proximal tibia during landing ${ }^{6}$, suggesting that increased VGRF and PGRF in sports activities are risk factors for ACL injury. Landing with a flexed trunk and lower limbs is recommended to improve VGRF and PGRF control ${ }^{7)}$. In this landing posture with an extended trunk, the center of 

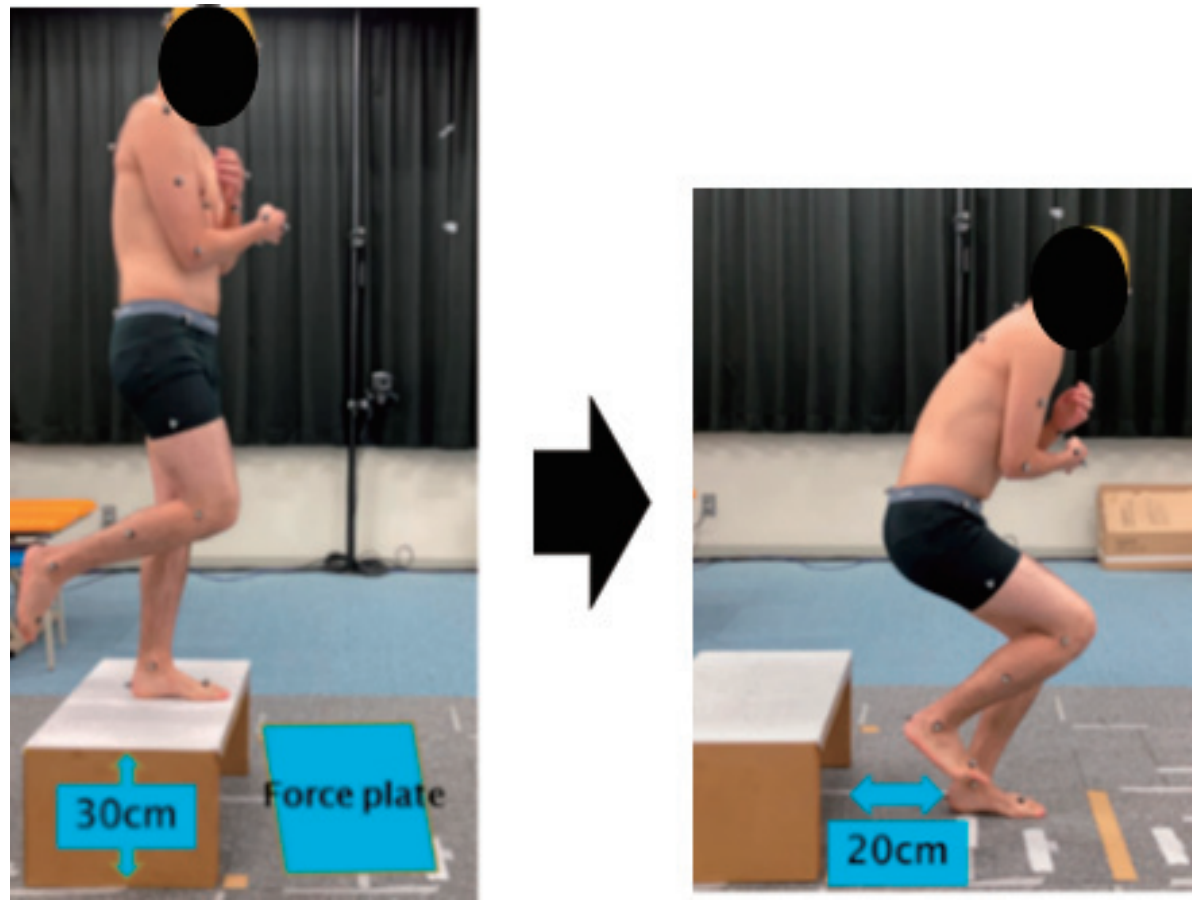

Fig. 1. One-legged landing motion measurement.

gravity is deflected backwards, and the PGRF and proximal tibia increase the forward withdrawal force.

The risks of ACL injury increase at the end of competition when fatigue accumulates ${ }^{8)}$. ACL and other sports injuries may also be caused by fatigue at the end of long games such as basketball and soccer ${ }^{8)}$. There is a difference between injuries during games and practice, with game injuries accounting for $66 \%$ of all injuries ${ }^{8)}$. When performing stop jumps before and after fatigue, there is an increase in peak anterior shear force and a decrease in knee flexion angle of approximately $14 \% \%^{9)}$. Oonishi et al. reported that fatigue from desk-based computational tasks can delay the switch between primary and antagonist muscles and reduce neuromuscular coordination during jumping, based on electromyography ${ }^{10)}$. However, fatigue during physical activity can be divided into peripheral and central factors, and central fatigue, including brain fatigue during exercise, can be explained by a decrease in brain glycogen ${ }^{11)}$. Such fatigue may affect dynamic muscle control, changes in movement patterns, and neuromuscular control. Most fatigue tasks were full-body exercise methods; landing movements after running or squatting movements ${ }^{9)}$. However, no studies have compared peripheral and central factors separately, and it is unclear what kind of fatigue causes changes in dynamic alignment during physical activity. Without identifying the type of fatigue, it is unlikely that specific injury prevention and conditioning methods can be proposed.

The present study aimed to investigate how lowerlimb muscle fatigue, cardiopulmonary fatigue, and brain fatigue tasks affect trunk and lower-limb alignment and GRF or time to peak vertical GRF (pVGRF) during a one-legged landing motion to inform primary and secondary ACL injury prevention.

\section{Methods}

The subjects were 12 men (mean age $23.3 \pm 2.9$ years, height $173.6 \pm 5.8 \mathrm{~cm}$, weight $65.9 \pm 8.3 \mathrm{~kg}$ ). The number of subjects was calculated using Gpower version 3.1.9.4 with an effect size of 0.5 and power of 0.8 . The exclusion criteria were as follows: (1) history of hip, knee, or ankle fracture or surgery; (2) hip, knee, and ankle joints below the reference range of motion specified by the Japanese Orthopedic Association $^{12)}$.

The subjects performed a one-legged landing motion from a $30-\mathrm{cm}$ platform before and after fatigue tasks. The one-legged landing motion was performed with the lower limb on the kicking side (Fig. 1). Fatigue tasks included lower-limb muscle fatigue, cardiopulmonary fatigue, and brain fatigue tasks (Fig. 2). To assess the degree of fatigue, a Borg scale was used during the motor and brain fatigue tasks, and salivary amylase levels were measured before and after the tasks using a salivary amylase sympathetic nerve monitor (Cocoro Meter, NIPRO Corporation, Osaka, Japan). Salivary amylase levels are elevated in response to mental and physical stress and are an excellent indicator when examining both types of stress ${ }^{13)}$. Subjects were asked to refrain from drinking anything other than water for $2 \mathrm{~h}$ prior to saliva collection, following the method of Yamaguchi et $\mathrm{al}^{14)}$. For measurement of joint angles and moments and GRF, a three-dimensional (3D) motion analysis device (Vicon Nexus, Vicon, Hauppauge, NY, USA) consisting of 


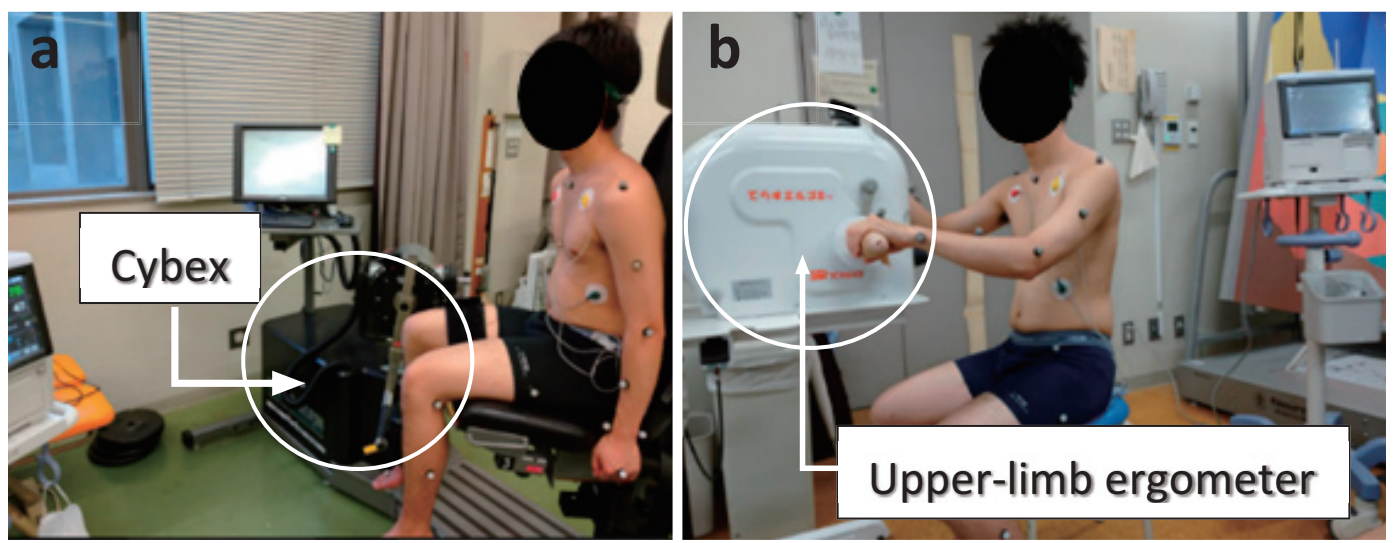

Fig. 2. Fatigue task implementation scene

a: Cybex implementation scene, b: Ergometer implementation scene

eight infrared cameras and a floor reaction-force meter (AMTI, Kanagawa, Japan) were used. The sampling frequency was set to $100 \mathrm{~Hz}$ for the infrared camera and 1,000 $\mathrm{Hz}$ for the floor reaction-force meter. The subjects wore sports spats and were measured barefoot. The Plug-in Gait Full Body AI model (Vicon) records 39 sites across the body with infrared reflective markers $14 \mathrm{~mm}$ in diameter. To ensure reproducibility of measurement, the joint angles in the static standing position before the measurement were checked, and the same examiner always wore the marker. The present study used this model to record the trunk and lower-limb joint angles and moments during the one-legged landing motion using a 3D motion analysis device.

Subjects were instructed to stand on one leg on the front side of a 30-cm platform, cross the upper limbs at the anterior chest, jump and land on one leg facing $20 \mathrm{~cm}$ in front of the platform, and remain still for $3 \mathrm{~s}$ after landing. They were instructed to look forward as much as possible (Fig. 1). If subjects could not avoid movement for $3 \mathrm{~s}$ after landing, moved the foot after landing, or moved the hands away from the chest, it was considered a failed trial. Three successful trials were recorded. There were no breaks between trials. The success data obtained for each of the three times before and after the task were analyzed with NEXUS version 2.8 (Vicon).

Initial grounding was defined as the point at which the vertical floor reaction force exceeded $10 \mathrm{~N}$. The 3D motion analyzer measured the hip and knee/ankle joint angles and knee valgus angles at the pVGRF, the trunk flexion angle at the peak posterior GRF (pPGRF), and the maximum joint moments of hip and knee extension and ankle plantarflexion. The trunk flexion angle was calculated as the angle of the thorax in spatial coordinates. For the joint moment, in addition to measuring the maximum value, the integral value was calculated using the interval from the initial ground contact to the peak value as the integration interval. The GRF parameters selected were pVGRF, pPGRF, the time taken from initial ground contact to $\mathrm{pVGRF}$ (time to
pVGRF), and loading rate (pVGRF/time to pVGRF), which indicates the impact absorption. The GRF was standardized by weight.

\section{Fatigue tasks}

The following fatigue tasks were conducted on different days. Each task was conducted more than one week after the previous experiment. In addition, each task was measured in random order. During each fatigue task, subjects were instructed to keep the trunk in the middle position. A total of 12 subjects performed all the following tasks.

\section{Lower-limb muscle fatigue task (motor task)}

We used a muscle function analysis exercise device (Cybex NORM, Lumex, Carol Stream, IL, USA) (Fig. 2) to move the leg muscles by performing isokinetic contractions with an angular velocity of $180^{\circ}$ /s over a range of motion of $90^{\circ}$ to $0^{\circ}$ at the knee joint for $10 \mathrm{~min}$. This movement was performed on the leg on the side of landing movement. To check the subjective level of fatigue, the Borg scale was constantly displayed, and a score of 13-14 was set as the target intensity. The heart rate and respiratory rate were monitored to ensure that they did not exceed $100 \mathrm{bpm}$ (tachycardia) or 25 breaths/min (tachypnea), respectively.

\section{Cardiopulmonary fatigue task (motor task)}

We performed a 10-min movement using an upperextremity ergometer (Terasu-ergoIII, Showa Denki, Osaka, Japan) (Fig. 2) to perform cardiopulmonary movements. To allow the movement load to be set at an aerobic level, the target heart rate during movement was set at approximately $70 \%$ of the predicted maximum heart rate (220-age) based on data from the American College of Sports Medicine ${ }^{15}$. Heart rate was monitored during movement using electrocardiogram pads worn on the chest. An upper-limb ergometer is a device for improving muscle strength and endurance by driving the upper limb, and the exercise was performed 
Table 1. Degree of fatigue before and after each condition

\begin{tabular}{llcccc}
\hline & & $\begin{array}{c}\text { Lower-limb } \\
\text { muscle } \\
\text { fatigue group }\end{array}$ & $\begin{array}{c}\text { Cardiopulmonary } \\
\text { fatigue group }\end{array}$ & $\begin{array}{c}\text { Brain fatigue } \\
\text { group }\end{array}$ & $\begin{array}{c}\text { Control } \\
\text { group }\end{array}$ \\
\hline Salivary amylase levels (KU/L) & Pre & $22.4 \pm 9.8$ & $22.8 \pm 7.0$ & $20.4 \pm 6.6$ & $22.1 \pm 6.0$ \\
& Post & $49.0 \pm 16.2$ & $47.7 \pm 15.7$ & $46.1 \pm 16.6$ & $20.8 \pm 6.3$ \\
& Diff & $26.6 \pm 14.3^{* *}$ & $24.8 \pm 13.1 * *$ & $25.7 \pm 13.7 *$ & $-1.3 \pm 3.5$ \\
Borg scale scores & Pre & $7.0 \pm 0.0$ & $7.0 \pm 0.0$ & $7.0 \pm 0.0$ & $7.0 \pm 0.0$ \\
& Post & $13.0 \pm 1.3$ & $13.0 \pm 0.6$ & $13.0 \pm 0.5$ & $7.0 \pm 0.0$ \\
& Diff & $6.0 \pm 1.2^{*}$ & $6.2 \pm 0.6^{*}$ & $5.5 \pm 0.5^{*}$ & $0.0 \pm 0.0$ \\
\hline
\end{tabular}

$*: p<0.05$ vs. control group, ${ }^{* *}: p<0.01$ vs. control group, Diff: difference

by grasping a handle and rotating it in the sitting position.

\section{Brain fatigue task (desk task)}

The Uchida-Kraepelin test (Japan Psychiatric Technology Institute Inc.) was performed for $10 \mathrm{~min}$. The UchidaKraepelin test a psychological test that repeatedly adds single digits and captures personality and behavioral characteristics from the total amount of work and incorrect answers. It was also used as a brain fatigue task in a previous study $^{10)}$

\section{Control task}

Subjects during the control task remained in a sitting position for $10 \mathrm{~min}$.

\section{Data analysis}

The results were checked for normality by the Kolmogorov-Smirnov test, taking the difference between the pre- and post-task values for each task. Non-normally distributed values were subjected to the Friedman test to compare differences between the four tasks. When significant differences were identified, Bonferroni's adjustment was performed with the Wilcoxon signed-rank sum test as a multiple comparison test and the differences between the pre- and post-task values for each task were compared. The differences between the four conditions before fatigue were tested in the same way. Statistical analysis was conducted using EZR version $1.52^{16)}$, with all levels of significance set at $5 \%$. This study was conducted with the approval of the Research Ethics Committee of Arakawa Campus, Tokyo Metropolitan University (approval number: 19073). In accordance with the Declaration of Helsinki, subjects were given a written explanation of the study and consent was obtained

\section{Results}

Pre- and post-task differences in salivary amylase levels and Borg scale scores (Table 1)

A maximum of 5 trials of the landing movement were performed, and the 3 successful trials were analyzed. Re- sults of the analysis of the landing movements before fatigue for each item showed no significant differences between the conditions.

There were significant differences in salivary amylase levels and Borg scale scores. There were also significant differences in salivary amylase levels within the lower-limb muscle fatigue, cardiopulmonary fatigue, and brain fatigue tasks, with higher values after each task compared to the control task, but there was no significant difference between the tasks. The Borg scale scores showed significant differences among the lower-limb muscle fatigue, cardiopulmonary fatigue, and brain fatigue tasks, with higher values after each task compared to the control task. No significant differences were found between the other tasks.

\section{Pre- and post-task differences in joint angles (Table 2)}

There was a significant difference in trunk flexion angle at pPGRF, but no significant differences in the other items. There were also significantly lower values for the trunk flexion angle at pPGRF in the brain fatigue task than in the control task. There was no significant difference in the trunk flexion angle between the other tasks.

Pre-and post-task differences in lower-limb joint moments (Table 3)

No significant differences were noted between tasks in the maximum joint moment or integral values after landing on one leg for the hip, knee, and ankle joints.

\section{Pre- and post-task differences in GRF (Table 3)}

There was a significant difference in time to pVGRF, but no significant difference was found in pVGRF, loading rate, and pPGRF. Time to pVGRF was significantly shorter in the lower-limb muscle fatigue task than in the control task. No significant differences were found between the other tasks.

\section{Discussion}

Regarding the relationship between fatigue and landing motion, drop jumps after fatigue increased the knee ab- 
Table 2. Lower-extremity joint angles and trunk angle before and after each condition

\begin{tabular}{llcccc}
\hline & & $\begin{array}{c}\text { Lower-limb } \\
\text { muscle } \\
\text { fatigue group }\end{array}$ & $\begin{array}{c}\text { Cardiopulmonary } \\
\text { fatigue group }\end{array}$ & $\begin{array}{c}\text { Brain fatigue } \\
\text { group }\end{array}$ & $\begin{array}{c}\text { Control } \\
\text { group }\end{array}$ \\
\hline Hip flexion at $\mathrm{pVGRF}$ timepoints $\left(^{\circ}\right)$ & Pre & $25.7 \pm 6.7$ & $26.8 \pm 8.6$ & $26.0 \pm 6.7$ & $27.7 \pm 6.0$ \\
& Post & $26.8 \pm 8.4$ & $27.3 \pm 8.7$ & $24.1 \pm 6.3$ & $26.2 \pm 6.8$ \\
Knee flexion at $\mathrm{pVGRF}$ timepoints $\left(^{\circ}\right)$ & Diff & $1.1 \pm 6.0$ & $0.5 \pm 2.5$ & $-2.0 \pm 2.4$ & $-1.5 \pm 1.8$ \\
& Pre & $37.6 \pm 6.2$ & $35.3 \pm 6.6$ & $35.3 \pm 6.3$ & $39.5 \pm 6.0$ \\
& Post & $38.0 \pm 6.1$ & $36.2 \pm 6.3$ & $35.2 \pm 6.2$ & $39.2 \pm 7.2$ \\
Knee valgus at $\mathrm{pVGRF}$ timepoints $\left(^{\circ}\right)$ & Diff & $0.4 \pm 5.3$ & $0.9 \pm 1.9$ & $0.0 \pm 2.3$ & $-0.3 \pm 2.5$ \\
& Pre & $7.1 \pm 9.6$ & $6.5 \pm 5.3$ & $7.3 \pm 4.6$ & $6.0 \pm 6.6$ \\
Ankle dorsiflexion at $\mathrm{pVGRF}$ timepoints $\left(^{\circ}\right)$ & Pre & $15.2 \pm 3.8$ & $16.0 \pm 4.5$ & $15.3 \pm 5.4$ & $17.7 \pm 5.0$ \\
& Post & $6.6 \pm 9.1$ & $5.3 \pm 5.9$ & $7.0 \pm 4.6$ & $5.0 \pm 7.3$ \\
& Post & $15.1 \pm 4.5$ & $15.7 \pm 4.7$ & $15.5 \pm 5.4$ & $17.9 \pm 5.4$ \\
Trunk flexion at $\mathrm{pPGRF}$ timepoints $\left(^{\circ}\right)$ & Diff & $-0.1 \pm 3.1$ & $-0.2 \pm 1.4$ & $0.2 \pm 2.0$ & $0.1 \pm 1.9$ \\
& Pre & $13.7 \pm 5.9$ & $14.6 \pm 7.1$ & $14.7 \pm 9.1$ & $13.5 \pm 7.8$ \\
& Post & $13.8 \pm 5.6$ & $14.2 \pm 7.1$ & $9.2 \pm 7.8$ & $14.4 \pm 8.3$ \\
& Diff & $0.1 \pm 4.0$ & $-0.4 \pm 2.3$ & $-5.5 \pm 6.9 *$ & $0.9 \pm 2.8$ \\
\hline
\end{tabular}

$*: p<0.05$ vs. control group, Diff: difference, pVGRF: peak vertical ground reaction force, pPGRF: peak posterior ground reaction force

duction moment ${ }^{17)}$. After performing unexpected runningstop movements during a fatigue task including squats and step-up movements, a decrease in knee flexion angle after fatigue was observed ${ }^{18)}$. However, the effect of fatigue factors on landing movements is still unclear, and this study examined the differences in fatigue tasks.

No significant differences in salivary amylase levels and Borg scale scores were observed among the tasks, suggesting that the stress load levels were equivalent; salivary amylase levels after each task were significantly higher than those in the control task. Previous studies have suggested an association between salivary amylase levels and general fatigue and stress ${ }^{19)}$. Our results suggest that the participants were able to load a certain amount of physical and mental stress for each 10-min task. Each task had a higher Borg scale score than the control task, and subjective fatigue was also recorded.

The brain fatigue task led to a significantly lower trunk flexion angle at pPGRF than the control task. However, there was no significant difference between the fatigue task groups, suggesting that the brain fatigue task may affect the landing strategy compared to the resting state. Ohji et al. found a negative correlation between PPGRF and the angle of trunk flexion during one-legged landing ${ }^{20)}$. In this study, the trunk flexion angle of the landing motion was decreased in the brain fatigue task. Thus, the center of gravity of the body and the point of load on the foot is shifted backward. Although the present study found no changes in pPGRF, the reduction in trunk flexion angle observed due to brain fatigue caused by the brain fatigue task may be more pronounced during overlapping brain exhaus- tion during competition. Inoue showed that fatigue due to central exhaustion can cause a decrease in spontaneous behaviors, which may have caused the subjects to insufficient posture formation to adapt to the environment, such as landing movements ${ }^{21)}$. Compared to the neutral trunk position in static standing, a trunk flexion posture that promotes shock absorption during landing seems to require active postural changes. Therefore, a possible reason for the decrease in trunk flexion angle in this study could be that posture formation was insufficient due to brain fatigue. In monkeys, brain fatigue from a simple visual response task resulted in a delay in reaction time ${ }^{22)}$. Fatigue during the computational task may delay switching between the main actuators and antagonists and reduce neuromuscular coordination during a jumping task, based on electromyographic examination of the quadriceps and biceps femoris muscles after a 15 -min Uchida-Kraepelin test ${ }^{10)}$. Therefore, in the present study, the brain fatigue task with the 10-min Uchida-Kraepelin test may have an altered muscle response. Central fatigue, including cerebral fatigue during exercise, may also be explained by a decrease in glycogen in the brain ${ }^{11)}$. The delayed reaction time, distraction, and changes in neuromuscular coordination caused by brain fatigue may have led to changes in postural strategies before and after landing, decreasing the trunk flexion angle. Neuromuscular coordination changes due to brain fatigue can delay the switching time of antagonist muscles ${ }^{10)}$, and if the same occurred in the antagonist muscles of the trunk and hip periarticular muscles, it may have affected postural control. However, since no brain function or exercise physiology tests were conducted in this study, it is unclear 
Table 3. Maximum lower-limb joint moments and GRF parameters before and after each condition

\begin{tabular}{|c|c|c|c|c|c|}
\hline & & $\begin{array}{l}\text { Lower-limb } \\
\text { muscle } \\
\text { fatigue group }\end{array}$ & $\begin{array}{l}\text { Cardiopulmonary } \\
\text { fatigue group }\end{array}$ & $\begin{array}{l}\text { Brain fatigue } \\
\text { group }\end{array}$ & Control group \\
\hline \multirow[t]{3}{*}{ Hip M. (Nm/kg) } & Pre & $0.356 \pm 0.286$ & $0.338 \pm 0.297$ & $0.394 \pm 0.245$ & $0.345 \pm 0.129$ \\
\hline & Post & $0.299 \pm 0.174$ & $0.369 \pm 0.261$ & $0.337 \pm 0.190$ & $0.341 \pm 0.151$ \\
\hline & Diff & $-0.057 \pm 0.290$ & $0.031 \pm 0.109$ & $-0.058 \pm 0.143$ & $-0.003 \pm 0.115$ \\
\hline \multirow[t]{3}{*}{ Knee M. (Nm/kg) } & Pre & $0.088 \pm 0.052$ & $0.124 \pm 0.067$ & $0.101 \pm 0.055$ & $0.101 \pm 0.049$ \\
\hline & Post & $0.083 \pm 0.055$ & $0.102 \pm 0.040$ & $0.098 \pm 0.051$ & $0.104 \pm 0.053$ \\
\hline & Diff & $-0.006 \pm 0.058$ & $-0.022 \pm 0.060$ & $-0.003 \pm 0.028$ & $0.003 \pm 0.045$ \\
\hline \multirow[t]{3}{*}{ Ankle M. (Nm/kg) } & Pre & $0.006 \pm 0.006$ & $0.008 \pm 0.007$ & $0.007 \pm 0.007$ & $0.008 \pm 0.006$ \\
\hline & Post & $0.006 \pm 0.010$ & $0.005 \pm 0.004$ & $0.006 \pm 0.005$ & $0.009 \pm 0.011$ \\
\hline & Diff & $0.000 \pm 0.010$ & $-0.003 \pm 0.006$ & $-0.001 \pm 0.004$ & $0.001 \pm 0.005$ \\
\hline \multirow[t]{3}{*}{ Hip M. integral (Nm/kg) } & Pre & $-3.805 \pm 1.644$ & $-3.837 \pm 1.405$ & $-3.870 \pm 1.463$ & $-3.575 \pm 1.564$ \\
\hline & Post & $-3.922 \pm 1.386$ & $-3.605 \pm 1.609$ & $-4.045 \pm 1.371$ & $-3.624 \pm 1.279$ \\
\hline & Diff & $-0.118 \pm 0.770$ & $0.233 \pm 0.767$ & $-0.175 \pm 0.432$ & $-0.049 \pm 1.097$ \\
\hline \multirow[t]{3}{*}{ Knee M. integral (Nm/kg) } & Pre & $0.038 \pm 0.242$ & $0.100 \pm 0.254$ & $0.091 \pm 0.284$ & $-0.023 \pm 0.313$ \\
\hline & Post & $0.071 \pm 0.254$ & $0.107 \pm 0.187$ & $0.089 \pm 0.212$ & $-0.050 \pm 0.313$ \\
\hline & Diff & $0.033 \pm 0.162$ & $0.007 \pm 0.143$ & $-0.002 \pm 0.112$ & $-0.027 \pm 0.133$ \\
\hline \multirow[t]{3}{*}{ Ankle M. integral (Nm/kg) } & Pre & $-0.195 \pm 0.034$ & $-0.188 \pm 0.042$ & $-0.184 \pm 0.039$ & $-0.178 \pm 0.044$ \\
\hline & Post & $-0.179 \pm 0.057$ & $-0.184 \pm 0.036$ & $-0.170 \pm 0.040$ & $-0.185 \pm 0.045$ \\
\hline & Diff & $0.016 \pm 0.051$ & $0.004 \pm 0.025$ & $0.014 \pm 0.024$ & $-0.007 \pm 0.030$ \\
\hline \multirow[t]{3}{*}{ pVGRF (\%BW) } & Pre & $46.5 \pm 9.1$ & $50.2 \pm 9.8$ & $47.9 \pm 9.9$ & $45.2 \pm 11.7$ \\
\hline & Post & $45.8 \pm 10.7$ & $49.0 \pm 11.6$ & $50.8 \pm 10.8$ & $45.1 \pm 12.0$ \\
\hline & Diff & $-0.6 \pm 7.2$ & $-1.1 \pm 4.7$ & $2.9 \pm 5.4$ & $-0.1 \pm 3.5$ \\
\hline \multirow[t]{3}{*}{ Time to pVGRF (ms) } & Pre & $58.3 \pm 10.7$ & $53.7 \pm 9.5$ & $55.5 \pm 13.1$ & $56.9 \pm 13.0$ \\
\hline & Post & $54.0 \pm 10.8$ & $53.8 \pm 11.0$ & $53.4 \pm 10.2$ & $56.6 \pm 13.7$ \\
\hline & Diff & $-4.3 \pm 2.9 *$ & $0.1 \pm 3.5$ & $-2.1 \pm 5.1$ & $-0.3 \pm 2.3$ \\
\hline \multirow[t]{3}{*}{ Loading rate $(\% \mathrm{BW} / \mathrm{ms})$} & Pre & $0.9 \pm 0.3$ & $1.0 \pm 0.3$ & $0.9 \pm 0.4$ & $0.9 \pm 0.4$ \\
\hline & Post & $0.9 \pm 0.4$ & $1.0 \pm 0.4$ & $1.0 \pm 0.4$ & $0.9 \pm 0.4$ \\
\hline & Diff & $0.1 \pm 0.2$ & $0.0 \pm 0.2$ & $0.1 \pm 0.2$ & $0.0 \pm 0.1$ \\
\hline \multirow[t]{3}{*}{ pPGRF (\%BW) } & Pre & $-5.8 \pm 1.2$ & $-6.1 \pm 0.9$ & $-6.1 \pm 1.2$ & $-6.1 \pm 1.4$ \\
\hline & Post & $-5.9 \pm 1.2$ & $-6.2 \pm 1.3$ & $-6.2 \pm 1.1$ & $-6.2 \pm 1.3$ \\
\hline & Diff & $0.0 \pm 0.6$ & $-0.1 \pm 0.6$ & $-0.1 \pm 0.6$ & $-0.1 \pm 0.3$ \\
\hline
\end{tabular}

*: $p<0.05$ vs. control group

Diff: difference; Hip M.: hip moment; Knee M.: knee moment; Ankle M.: ankle moment; pVGRF: peak vertical ground reaction force; Loading rate: $\mathrm{pVGRF/time} \mathrm{to} \mathrm{pVGRF}$; $\mathrm{pPGRF}$ : peak posterior ground reaction force, BW: body weight

whether the brain fatigue task reduced neuromuscular coordination or brain glycogen. Although previous studies described the change in muscle reaction time in brain fatigue and the increase of anterior withdrawal force and ACL stress of the tibia with a decrease in trunk flexion angle, no study has focused on the trunk flexion angle in the landing motion before and after fatigue. The decrease of trunk flexion angle in the landing motion may be a factor of increased ACL stress due to the backward shift of the center of gravity. Therefore, it is important to consider that landing strategies can change during brain fatigue when compared to resting conditions.

The time to pVGRF was significantly shorter in the lower-limb muscle fatigue task than in the control task. Fatigue is an exogenous factor affecting the skeletal muscula- ture and nervous system and is associated with decreased joint awareness and increased joint laxity ${ }^{23)}$. Fatigued muscle fibers exhibit a reduced capacity to absorb energy and an increased anterior deviation of the tibia from altered neuromuscular function ${ }^{24,25)}$. We are unable to describe the factors that contributed to the shortened time to pVGRF: there were no significant differences in joint range of motion or joint moment, and articulation, neuromuscular function, and tibial mobility were not measured. The shortened time to pVGRF indicated that the time to reach the maximum vertical floor reaction force after landing on one leg was shortened. Therefore, it is possible that in the lowerlimb muscle fatigue group, the landing was such that the floor was slammed against their feet into the floor upon landing. It has been suggested that during such landing 
movements, the quadriceps muscle contracts more strongly, leading to increased anterior tibial translation ${ }^{6}$. There was no significant change in pVGRF. This may be due to the fact that changes in the fatigue task load and time to pVGRF were minor. The results of this study showed that the time to pVGRF was shorter in the leg-muscle fatigue group, suggesting that the risk of ACL injury may be increased during leg-muscle fatigue, at least when compared to rest.

The range of motion, joint moment, and floor reaction force did not change because the loading was from a light motor task (Borg scale score $=13$ ). Analysis of the lowerextremity joint angle and moment during landing during the stop-jumping motion after the fatigue protocol found that the knee flexion angle decreased by $3.5^{\circ}$ and the hip flexion angle decreased by $7.7^{\circ}$, but there was no significant difference in the knee joint moment ${ }^{26)}$. The kinematics of the landing motion were analyzed by assuming the fatigue task before the one-legged landing motion as the maximum number of repetitions (Borg scale score $=18$ ), or $85 \%$ of the predicted maximum heart rate $^{26}$. Considering the minor changes in the lower-limb joint angle and floor reaction force values during the landing motion at near-maximal fatigue, it is reasonable to assume that the fatigue task in this study did not result in a significant change. However, one limitation of this study is the possibility of inducing ACL damage during landing movements when the task load is set to the maximum load, which makes it difficult to use in research.

Sporting games and practices last longer than the tasks in this study, and fatigue is expected to accumulate in the last stage. Time to pVGRF during lower-extremity muscle fatigue and trunk flexion angle during brain fatigue, which were altered during the 10-min task and prior to reaching maximal fatigue, may be more pronounced during such actual sports activities. Simply rinsing out the mouth with a sugar solution as a preventive measure against central fatigue has been shown to improve performance ${ }^{27)}$. The results of this study indicate that the landing motion may be altered during fatigue compared to resting, which may provide important basic information for studying preventive measures. Research into strategies to eliminate brain fatigue and lower-limb muscle fatigue is necessary.

\section{Conclusion}

In this study, the kinematics and floor reaction forces of the trunk and lower limbs during a one-legged landing motion were measured using a 3D motion analysis system and GRF meter before and after lower-limb muscle fatigue, cardiopulmonary fatigue, brain fatigue, and the control task, and their differences were analyzed. The maximum trunk flexion angle on landing on one leg was significantly lower in the brain fatigue task than in the control task. The time to pVGRF was significantly shorter in the lower-limb muscle fatigue task than in the control task. No significant differences were obtained among the fatigue task groups. Even if the fatigue task is relatively minor, the effects of muscle and brain fatigue in the lower extremities may change the strategy during the landing movement when compared to the resting state.

Conflict of Interest: The authors declare no conflicts of interest related to this study.

\section{References}

1) Hewett TE, Lindenfeld TM, et al.: The effect of neuromuscular training on the incidence of knee injury in female athletes. A prospective study. Am J Sports Med. 1999; 27: 699-706.

2) Maletius W and Messner K: Eighteen- to twenty-four-year follow-up after complete rupture of the anterior cruciate ligament. Am J Sports Med. 1999; 27: 711-717.

3) Boden BP, Dean GS, et al.: Mechanisms of anterior cruciate ligament injury. Orthopedics. 2000; 23: 573-578.

4) Noyes TE, Barber-Westin SD, et al.: The drop-jump screening test: difference in lower limb control by ender and effect of neuromuscular training in female athletes. Am J Sports Med. 2005; 33: 197-207.

5) Hewett TE, Myer GD, et al.: Biomechanical measures of neuromuscular control and valgus loading of the knee predict anterior cruciate ligament injury risk in female athletes: a prospective study. Am J Sports Med. 2005; 33: 492-501.

6) $\mathrm{Yu} \mathrm{B}$, Lin CF, et al.: Lower extremity biomechanics during the landing of a stop-jump task. Clin Biomech. 2006; 21: 297-305.

7) Blackburn JT and Padua DA: Sagittal-plane trunk position, landing forces, and quadriceps electromyographic activity. J Athl Train. 2009; 44: 174-179.

8) Hawkins RD, Hulse MA, et al:: The association football medical research programme: an audit of injuries in professional football. Br J Sports Med. 2001; 35: 43-47.

9) Chappell JD, Herman DC, et al.: Effect of fatigue on knee kinetics and kinematics in stop-jump tasks. Am J Sports Med. 2005; 33: 1022-1029.

10) Oonishi S, Hunasaki Y, et al.: Changes in nerve-muscle coordination caused by muscle and brain fatigue: analysis using the silent period of quadriceps and hamstrings. J Jpn Soc Clin Sports Med. 2018; 26: 236-241.

11) Matsui T and Soya H: Nou gurikogen no genshou to chuusuu-sei hirou (Brain glycogen depletion and central fatigue). J Health Phys Educ Recreat. 2010; 60: 797-804[in Japanese].

12) Yonemoto $K$, Ishigami $S$, et al.: Kansetsukadouiki-hyouji narabini sokuteihou. Jpn J Rehabil Med. 1995; 32: 207-217[in Japanese].

13) Nakano A and Yamaguchi M: Evaluation of human stress using salivary amylase. Jpn Soc Biofeedback Res. 2011; 38: 4-9.

14) Yamaguchi M, Hanawa N, et al:: Evaluation of a novel monitor for the sympathetic nervous system using salivary amylase activity. Trans Jpn Soc Med Biol Eng. 2007; 45: 161-168. 
15) American College of Sports Medicine: The recommended quantity and quality of exercise for developing and maintaining cardiorespiratory and muscular fitness, and flexibility in healthy adults. Med Sci Sports Exerc. 1998; 30: 975-991.

16) Kanda Y: Investigation of the freely available easy-to-use soft 'EZR' for medical statistics. Bone Marrow Transplantation. 2013; 48: 452-458.

17) McLean SG, Fellin RE, et al.: Impact of fatigue on gender-based high-risk landing strategies. Med Sci Sports Exerc. 2007; 39: 502-514.

18) Cortes N, Greska E, et al: : Knee kinematics is altered postfatigue while performing a crossover task. Knee Surg Sports Traumatol Arthrosc. 2014; 22: 2202-2208.

19) Tanaka $Y$ and Wakida $S$ : Stress to hirou no biomarkers (Biomarkers of stress and fatigue). Nippon Yakurigaku Zasshi. 2011; 137: 185-188[in Japanese].

20) Ohji S, Aizawa J, et al.: Correlations between pre-landing sagittal plane kinematics and posterior ground reaction force during single-leg anterior jump-landing. J Phys Ther Sci. 2017; 32 : 751-755.

21) Inoue K: Hirou-bussitsu toshiteno nounai-TGF- $\beta$. J Health Phys
Educ Recreat. 2010; 60: 819-823[in Japanese].

22) Onoe H: Tanjyun-shikaku-hannoukadai niyoru hirou no kyakkanteki hyouka (Evaluation of fatigue using a simple reaction task in the macaque monkey). J Clin Exp Med. 2003; 204: 371376[in Japanese].

23) Rozzi SL, Lephart SM, et al.: Effects of muscular fatigue on knee joint laxity and neuromuscular characteristics of male and female athletes. J Athl Train. 1999; 34: 106-114.

24) Lepers RO, Hausswirth CH, et al.: Evidence of neuromuscular fatigue after prolonged cycling exercise. Med Sci Sports Exerc. 2000; 32: 1880-1886.

25) Wojtys EM, Wylie BB, et al.: The effects of muscle fatigue on neuromuscular function and anterior tibial translation in healthy knees. J Sports Med. 1996; 24: 615-621.

26) Cortes N, Quammen D, et al.: A functional agility short-term fatigue protocol changes lower extremity mechanics. J Sports Sci. 2012; 30: 797-805.

27) Carter JM, Jeukendrup AE, et al.: The effect of carbohydrate mouth rinse on 1-h cycle time trial performance. Med Sci Sports Exerc. 2004; 36: 2107-2111. 